\title{
Tumor miofibroblástico inflamatorio de arteria carótida interna resecado con embolización selectiva previa. Reporte de caso
}

\author{
Inflammatory myofibroblastic tumor related to the internal carotid artery, \\ extirpated after an arterial selective embolization
}

Jaime Bermeo S ${ }^{1,2}$, Cristián Bachelet R³, Felipe Cardemil M², Rodrigo Muñoz S².

\begin{abstract}
RESUMEN
El tumor miofibroblástico inflamatorio es una lesión infrecuente, cuya importancia radica en ser diagnóstico diferencial de otras neoplasias. Se le ha relacionado con infecciones, traumas, cirugías previas e inmunosupresión, pero se cree que éstos sólo serían el evento inicial que daría lugar a una serie de cascadas autoinmunes que perpetuarían al cuadro. Afecta a población infantil y adulta joven, con una edad media de aparición de 10 años, sin distinción de género. A continuación se presenta el caso de una mujer de 45 años de edad con diagnóstico de tumor miofibroblástico inflamatorio de arteria carótida interna, resecado con embolización arterial selectiva previa. Además, se revisa la literatura al respecto.
\end{abstract}

Palabras clave: Tumor miofibroblástico, resección, embolización arterial selectiva.

\begin{abstract}
The inflammatory myofibroblastic tumor is a rare condition, and its' relevant for being a differential diagnosis of many other neoplasms. It has been related with different infections, traumas, previous surgeries and immune deficiencies, but there are studies that support the theory that these factors are only the initial event of an autoimmune reaction that perpetuates the disease. It affects childs and young adults, with an average of 10 years old at the moment of presentation, without differences between genre. In this case, we report a 45 year old woman, with an histological diagnosis of inflammatory myofibroblastic tumor related to the internal carotid artery, extirpated after an arterial selective embolization. Besides, we review the literature.
\end{abstract}

Key words: Inflammatory pseudotumor, resection, arterial selective embolization.

\footnotetext{
Servicio de Otorrinolaringología, Hospital Carlos Van Buren, Valparaíso.

Escuela de Medicina, Universidad de Valparaíso.

3 Residente de Otorrinolaringología, Hospital del Salvador, Universidad de Chile.
} 


\section{INTRODUCCIÓN}

El tumor miofibroblástico inflamatorio (TMI) es una lesión infrecuente, cuya localización, tamaño y sintomatología son variables. Está compuesto por células miofibroblásticas fusiformes con una mezcla de células inflamatorias (linfocitos y células plasmáticas) y fibras colágenas. Su importancia radica como diagnóstico diferencial clínico, imagenológico e histopatológico de otras neopla$\operatorname{sias}^{1,2}$. Si bien la mayoría de los casos son de localización pulmonar y en población pediátrica, Ios pacientes con TMI de cabeza y cuello suelen ser adultos, hallándose frecuentemente en órbita y tracto aerodigestivo superior.

Se presenta el caso de una paciente adulta con diagnóstico de TMI de arteria carótida interna, siendo una localización poco usual según la literatura.

\section{CASO CLÍNICO}

Paciente de sexo femenino de 45 años de edad, con antecedente de diabetes mellitus insulinorrequirente secundaria a pancreatitis grave, vasculitis e hipotiroidismo. Derivada desde otro hospital de la red por cuadro de 5 meses de evolución caracterizado por odontalgia izquierda y disfonía. Se inicia su estudio por disfonía con nasofibroscopía, evidenciando parálisis de cuerda vocal izquierda. Continúa estudio con ecografía, tomografía computarizada de cuello y resonancia magnética (Figura 1), evidenciando masa cervical compatible con glomus carotídeo cervical izquierdo. Exámenes de laboratorio básicos resultan normales, a excepción de discreta leucocitosis. Se evalúa con neurorradiólogo y se decide realizar estudio angiográfico del territorio carotídeo bilateral incluyendo polígono de Willis, encontrando suficiencia del mismo. Se realiza embolización selectiva y sacrificio de la arteria carótida interna $(\mathrm{Cl})$, con resultado satisfactorio (Figura 2). Posteriormente se controla con angiorresonancia, objetivándose llene de las arterias del polígono de Willis a expensas de la carótida interna derecha y de la circulación posterior (Figura 3). A los 2 meses se realiza exéresis del tumor por abordaje cervical (Figura 4), resecándose una masa blanquecina de $8 \times 4 \mathrm{cms}$, adherida a la $\mathrm{Cl}$, vena yugular interna y planos adyacentes, sin adenopatías asociadas. Presenta buena evolución posoperatoria. La biopsia informa masa blanquecina fibrótica que engloba a $\mathrm{Cl}$ y a varios ganglios,

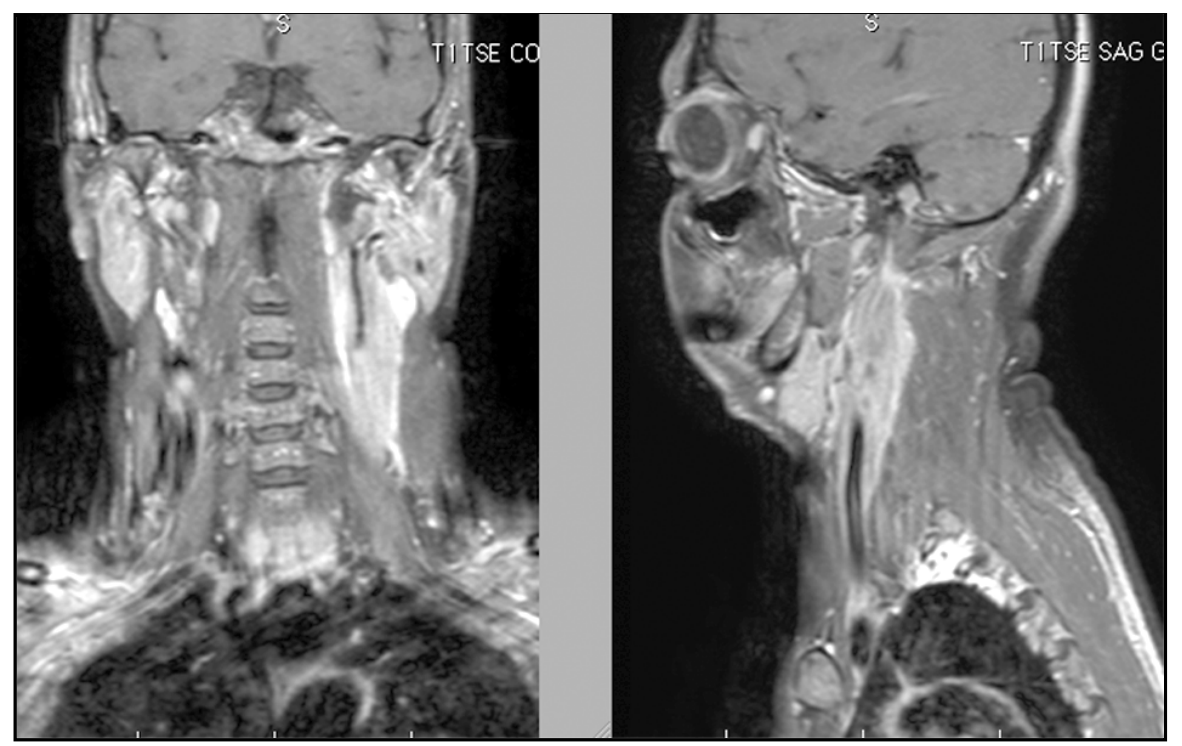

Figura 1. Corte coronal de resonancia magnética de encéfalo, en que se aprecia un proceso expansivo, invasivo de tejidos vecinos, en relación a la arteria carótida interna izquierda. 


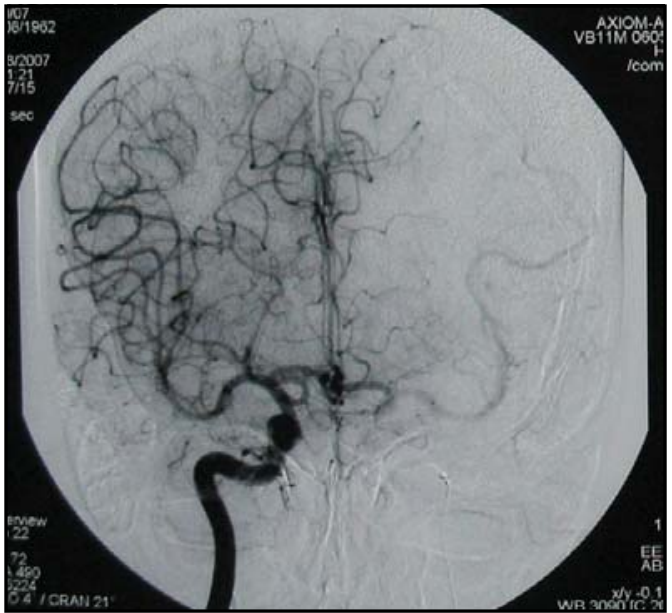

Figura 2. Angiografía cerebral 3D postembolización selectiva de arteria carótida interna izquierda que revela llene con medio de contraste desde la carótida derecha.

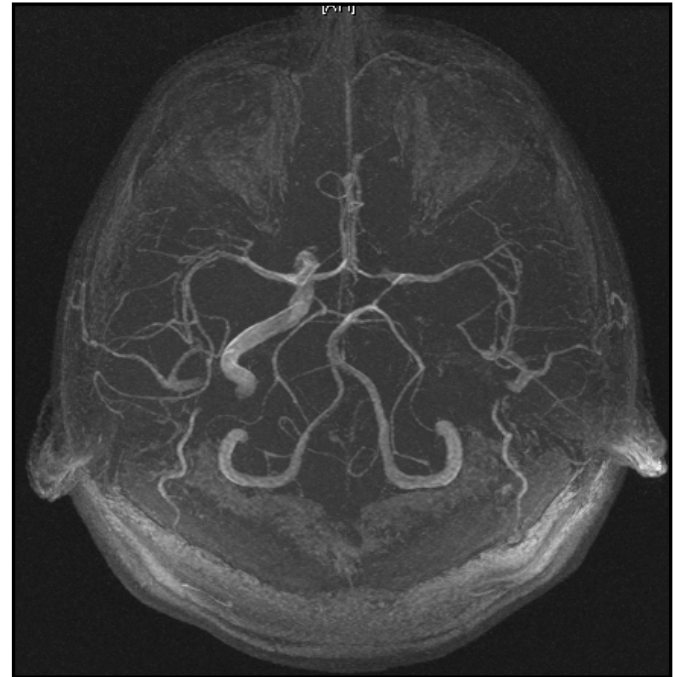

Figura 3. Corte axial de angiorresonancia de encéfalo posterior a embolización de arteria carótida interna en que se aprecia llene de las arterias del polígono de Willis a expensas de la carótida interna derecha y de la circulación posterior.

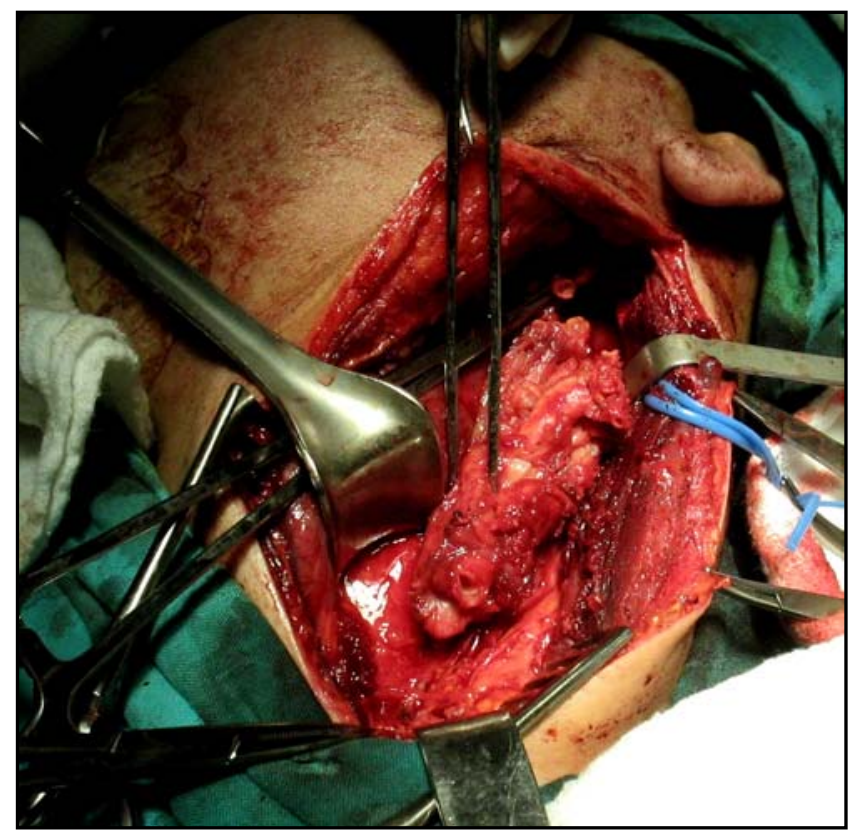

Figura 4. Abordaje cervical de exéresis quirúrgica de tumor miofibroblástico inflamatorio de arteria carótida interna.

compatible con tumor miofibroblástico inflamatorio (Figura 5), diagnóstico que se confirma con inmunohistoquímica. Este caso muestra una forma de presentación poco habitual del TMI, pero cumple con lo descrito para el diagnóstico.

\section{DISCUSIÓN}

El TMI ha recibido múltiples denominaciones en la literatura, entre las que destacan pseudotumor inflamatorio, granuloma de células plasmáticas, 


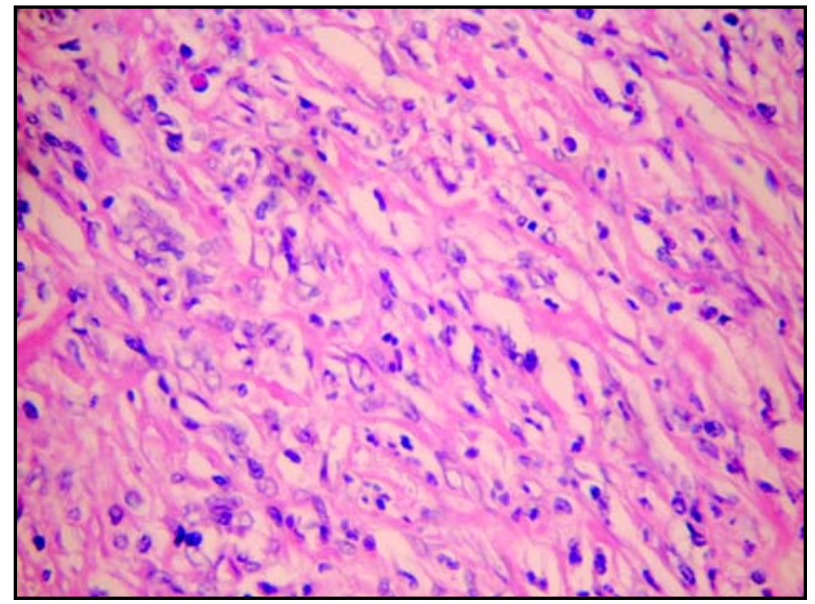

Figura 5. Corte histológico sobre tumor miofibroblástico inflamatorio en el que se aprecian miofibroblastos e infiltrado inflamatorio polimorfo sobre matriz colágena.

pseudotumor xantomatoso, complejo histiocitomacélula plasmática y proliferación miofibrohistiocítica inflamatoria². Fue descrito por Birch-Hirschfield en 1905 en un paciente con compromiso orbitario. Sin embargo, la primera serie importante fue reportada en 1973 por Bahadori y Liebow, identificando al TMI como el tumor pulmonar primario más frecuente en población pediátrica ${ }^{3-5}$.

Si bien se pensaba que era un proceso de naturaleza reactiva 0 inflamatoria exagerada, recientes investigaciones han demostrado recurrencia local y su asociación a alteraciones cromosómicas adquiridas ${ }^{2,6-8}$. Actualmente es considerado una neoplasia generalmente benigna con cierto potencial para transformación maligna (tumor borderline), siendo incluido desde el año 2005 en la clasificación OMS de tumores de partes blandas ${ }^{1}$. Se le ha relacionado con infecciones, traumas, cirugías previas, e inmunosupresión. Algunos reportes han descrito en estos tumores el hallazgo de secuencias de ADN de virus herpes humano 8 y sobreexpresión de interleuquina 6 y ciclina D17,9. Otros agentes infecciosos que han sido aislados son el virus Epstein-Barr, Mycoplasma sp, Nocardia sp y Actinomyces $s p^{2}$. Entre las alteraciones citogenéticas asociadas destaca la mutación del gen ALK, pero también se ha descrito afectación de los genes TPM3, RANBP2, CARS, CLTC y TPM $4^{1,10}$. Los intentos de identificar agentes etiológicos en el seno del TMI han tenido pobres resultados, por lo que se cree que su rol estaría restringido a la etapa inicial de la enfermedad, asociado a fenómenos autoinmunes posteriores. De este modo, se generaría una cascada de reacciones paracrinas en las cuales las células estromales y el entorno inflamatorio pudieran sufrir un intercambio de factores proinflamatorios y proliferantes, tras los cuales el tumor llegaría a ser autónomo ${ }^{2,9}$.

En general, el TMI es más frecuentemente encontrado en niños y adultos jóvenes, con una edad media de aparición de 10 años, sin distinción de género ${ }^{1,2}$. La principal localización es pulmonar, no obstante se ha descrito prácticamente en cualquier parte del cuerpo. A la fecha se han publicado alrededor de 3.000 casos a nivel mundial1,2,7,11. El subgrupo de TMI de cabeza y cuello afecta principalmente a población adulta, con una edad media de 59 años, ubicándose más frecuentemente en órbita, laringe, cavidad oral, amígdalas, espacio parafaríngeo, tracto sinonasal, glándulas salivales mayores y tráquea ${ }^{2,4}$. Son asintomáticos en el $50 \%$ de los casos, por lo que suelen presentarse como un incidentaloma. Su clínica es inespecífica, sobre todo en Ios TMI viscerales, pudiendo cursar con fiebre, sudoración nocturna, compromiso de estado general, pérdida de peso y adenopatías. También pueden presentarse síntomas locales, que dependerán de la ubicación de la lesión. Al laboratorio puede encontrarse aumento de la velocidad 
de eritrosedimentación, anemia microcítica hipocroma, trombocitosis e hipergamaglobulinemia policlonal ${ }^{2,11}$. Macroscópicamente suelen verse como lesiones nodulares o polipoides de color rojizo y superficie lisa, generalmente bien delimitadas en los casos pulmonares, pero en los TMI extrapulmonares pueden ser de límites menos definidos o multinodulares. Hasta en $10 \%$ pueden presentar pequeñas zonas de necrosis o calcificaciones en su espesor. En su perfil inmunohistoquímico tienen positividad difusa a la vimentina y expresión variable de actina 0 desmina. Las citoqueratinas son habitualmente negativas, aunque pueden presentarse alguna positividad focal. Para diferenciar de linfomas, el estudio inmunohistoquímico de subpoblaciones de linfocitos mostrará células T y B en el TMI ${ }^{1,2,6,11}$.

Su tratamiento está dado principalmente por la resección quirúrgica, aunque el uso de pulsos breves de corticoides en altas dosis ha tenido buena respuesta en reducir la masa tumoral y los síntomas $^{2,12}$. Pese a que la respuesta a corticoides es impredecible, en tumores irresecables o pacientes no aptos para cirugía han sido usados como terapia de elección. No obstante, siempre es deseable el manejo quirúrgico para comprobar la histología y disminuir la tasa de recidivas, ya que la reducción tumoral con corticoides no suele ser completa. Como alternativa a los corticoides en pacientes con contraindicación, la elección es radioterapia en dosis bajas. También se ha utilizado ciclosporina, clorambucil e indometacina. No existen datos definitivos de quimioterapia útil en TMI².

El pronóstico generalmente es bueno, aunque con una tasa de recurrencia que puede llegar hasta $25 \%$ en TMI extrapulmonares por las limitaciones ante su excisión, dado por los bordes poco definidos y gran adherencia a estructuras vitales ${ }^{1,2,11}$. La mortalidad específica es de menos de 10\%, producida principalmente por invasión local. Existen casos aislados de TMI viscerales que han originado metástasis, así como también otros de remisión espontánea. Todo esto indica la dificultad para predecir su comportamiento en base a su histología ${ }^{13,14}$. Entre los TMI de cabeza y cuello, son aún más raros los que comprometen la arteria carótida o la vaina carotídea, existiendo 4 casos reportados en la literatura. Se presentan como masa palpable o por dolor local y suelen estar muy adheridos a estructuras vecinas, por lo que se han intentado alternativas de manejo tanto de resección en bloque como de terapia médica exclusiva ${ }^{15-18}$, con resultados aún inciertos.

En este caso se realizó embolización previa de la arteria comprometida y luego resección quirúrgica. En controles alejados a 2 años no se ha objetivado recidiva local ni otras complicaciones sistémicas, lo que reafirma la utilidad del tratamiento quirúrgico como primera elección en estos pacientes.

\section{BIBLIOGRAFÍA}

1. Ereño C. La Nueva Clasificación de la O.M.S. 2005. Lesiones Precursoras y los Tumores de la Laringe, Hipofaringe y Tráquea. Rev Esp Patol 2007; 40(1): 3-10.

2. Das Narla L, Newman B, Spottswood SS, et al. Inflammatory Pseudotumor. RadioGraphics 2003; 23: 719-29.

3. De Vuysere S, Hermans R, Sciot R, et al. Extraorbital Inflammatory Pseudotumor of the Head and Neck: CT and MR Findings in Three Patients. Am J Neuroradiol 1999; 20: 1133-9.

4. HM Lee, Chol G, Chol CS, et AL. Inflammatory Pseudotumor of the Maxillary Sinus. Otolaryngol Head Neck Surg 2001; 125: 565-6.

5. Ma Y, Zieske A, Fenves A, et al. Bilateral Infiltrating Renal Inflammatory Pseudotumor Responsive to Corticosteroid Therapy. American Journal of Kidney Diseases 2008; 51 (1): 116-20.

6. Wu JM, Montgomery E. Classification and Pathology. Surg Clin N Am 2008; 88: 483-520.

7. Coffin CM, Watterson J, Priest JR, et al. Extrapulmonary inflammatory myofibroblastic tumor (inflammatory pseudotumor). A clinicopathologic and immunohistochemical study of 84 cases. Am J Surg Pathol 1995; 19: 859-72.

8. Lahat G, Lazar A, Lev D. Sarcoma epidemiology and etiology: potential environmental and genetic factors. Surg Clin N Am 2008; 88: 451-81.

9. Gómez-Roman JJ, Sánchez-Velasco P, Ocejo-Vinayals G, ET AL. Human herpesvirus-8 genes are expressed in pulmonary inflammatory myofibroblastic tumor (inflammatory pseudotumor). Am J Surg Pathol 2001; 25(5): 624-9. 
10. Chan JK, Cheuk W, Shimizu M. Anaplastic lymphoma kinase expression in inflammatory pseudotumors. Am J Surg Pathol 2001; 25(6): 761-8.

11. Husain AN, Kumar V. The Lung. Kumar: Robbins and Cotran: Pathologic Basis of Disease, $7^{\text {th }}$ ed. Elsevier Saunders 2005, Philadelphia, PA, USA.

12. Ceruse $P$, Ramade A, Vautrin R, et al. Inflammatory Pseudotumor of the Neck: A long-term result without surgical approach. Otolaryngol Head Neck Surg 2005; 132: 812-3.

13. Hagenstad CT, Kilpatrick SE, Pettenati MJ, et al. Inflammatory myofibroblastic tumor with bone marrow involvement. A case report and review of the literature. Arch Pathol Lab Med 2003; 127(7): 865-7.

14. Yamaguchi J, Sakamoto Y, Sano T, et al. Spontaneus regression of inflammatory pseudotumor of the liver: report of three cases. Surg Today 2007; 37(6): 525-9.

15. Farage L, Da Motta A, Goldenberg D, et al. Idiopathic inflammatory pseudotumor of the carotid sheath. Arq Neuropsiquiatr 2007; 65(4B): $1241-4$

16. KIm GE, Cho YP, Chung DH, et AL. Inflammatory pseudotumor of carotid artery: a case report. Cardiovasc Surg 1999; 7(3): 342-7.

17. LeE HK, Kim EJ, Ro JY, et AL. Inflammatory pseudotumor of the carotid artery: radiologic findings. J Comput Assist Tomogr 2003; 27(2): 253-6.

18. Окамото M, Takahashi $\mathrm{H}$, Yamanaka J, et al. Sclerosing inflammatory pseudotumor arising from the carotid artery region. Auris Nasus Larynx 1997; 24(3): 315-20. 